Rapid Reviews COVID-19

\title{
Review 2: "COVID-19 and (Un)Desired Fertility: The Effect of Stay-at-Home Orders on Abortions in Mexico City"
}

\section{Caitlin Gerdts 1}

${ }^{1}$ Vice President for Research, Ibis Reproductive Health, Research, USA

Published on: Nov 25, 2020

DOI: $10.1162 / 2 e 3983 f 5 . c 8813 b f c$

License: Creative Commons Attribution 4.0 International License (CC-BY 4.0). 


\section{$\underline{\text { RR:C19 Evidence Scale rating by reviewer: }}$}

- Misleading. Serious flaws and errors in the methods and data render the study conclusions misinformative. The results and conclusions of the ideal study are at least as likely to conclude the opposite of its results and conclusions than agree. Decision-makers should not consider this evidence in any decision.

$* * * * * * * * * * * * * * * * * * * * * * * * * * * * * * * * * * * * * * * *$

\section{Review:}

This paper presents data from government facilities providing abortion in Mexico City and a government-run hotline providing information about sexual and reproductive health topics. While the data are, indeed, a useful marker of the trends in services that the government is providing, the authors fail to account for a range of alternative explanations that could account for their findings, and thus, their conclusion that this study provides the "first empirical evidence anticipating the effects of COVID-19 and SAH on fertility" are easily challenged.

Broadly, the authors do not take into consideration the fact that misoprostol (a safe, WHO recommended abortifacient medication) is widely available in pharmacies in Mexico City, and there are many groups operating in and around the DF (and across the country) that provide information and support for people who are seeking to selfmanage abortions without clinical supervision/outside of the formal health system, and in some cases these groups may also facilitate access to abortifacient medications. The fact that the government ILE data reflects a decline in abortions performed is consistent with what we know about people's responses to medical settings in the COVID-19 pandemic-i.e. wanting to avoid enclosed spaces where people may be sick, as well as the fact that ILE services may be more time-consuming (including more time traveling and waiting at a clinic) than self-managing an abortion, which may be of particular concern now when people have small children at home and are navigating other economic/social constraints.

There is also the potential that, in cases where people do not know about safe abortifacient medications, they could attempt to self-induce abortion using unsafe methods. Not only do the authors not have data on abortions overall (both in and out of facility), they do not suggest these as an alternative explanation for the decline in abortion services, and immediately come to the conclusion that, based on an increase 
(though not a significant one given the wide confidence intervals) in pregnancy-related calls to the helpline, this must indicate an increase in fertility. Of course, an alternative explanation for the (possible) increase in pregnancy-related calls is that people may be more concerned about or have more questions about pregnancy in the midst of a pandemic than they did prior-this could be indicative of health concerns, a desire to get more information about labor and delivery services during COVID-19, etc., and not have anything at all to do with changes in fertility itself.

Before this paper is ready for publication, the authors need to address a number of additional factors that could influence their results and adjust their conclusions to better match the lived reality of people's reproductive lives in Mexico City during the pandemic.

A few specific comments below:

Page 4, paragraph 1: "this corresponds to 975 more unwanted pregnancies...". This is a baseless assumption (addressed above). The only thing that the authors can conclude is that this corresponds to 975 fewer abortions performed in government facilities. It is very possible that all, most, or at least some of those 975 people obtained abortions in other ways.

Page 4, paragraph 2: "whether the observed decline in abortions is due to women not being able to get one or due to women not wanting to get one under the new circumstances..." Again, this is missing a big piece about self-managed abortions, or abortions obtained outside of facilities (safe or otherwise).

Page 4 paragraph 2: "Our analysis shows evidence consistent with an increase in pregnancies following the start of SAH orders as well as important increases in domestic violence, in addition to fewer inquiries regarding contraception, arguably due to a loss of privacy." Again, a baseless assumption that needs to be corrected.

Page 4 paragraph 2 should read "likely due to restricted access as opposed to women being less likely to want to get an abortion" and the assumption needs correction based on the above explanations.

Page 10 para 2: "It is also likely that municipalities with higher COVID-19 mortality are more compliant with SAH orders." This is yet another assumption that is not based in evidence, in fact, we have seen consistently across the globe that places where 
COVID-19 mortality are highest are those with greater concentrations of poverty, people who are essential workers and are therefore more routinely exposed and who do not have the option to follow SAH orders. This needs to be corrected.

Page 13: "This result, taken together with the decline on abortions, presents us with suggestive evidence of an expected increase in fertility due to the pandemic". Again, as above people might be more concerned if they are pregnant, and have many questions that need answering that never needed answering before. The fact that there are huge confidence intervals makes the drawing of this conclusion quite reckless. 